\title{
Estudio de usabilidad y percepción de eficacia de los módulos que componen el
}

Programa Héroe

Study on Usability and Perception of Effectiveness in the Programa Hero Modules

BelÉN MESURADO*

Consejo Nacional de Investigaciones Científicas y Técnicas (CONICET) - Universidad Austral (Argentina) bmesurado@autral.edu.ar http://orcid.org/oooo-0002-5907-5854

\section{MARIANA FALCO}

LIDTUA - Consejo Nacional de Investigaciones Científicas y Técnicas (CONICET) - Universidad Austral (Argentina) MFalco@austral.edu.ar https://orcid.org/oooo-ooo2-0959-7435

\section{MARÍA JosÉ DistÉFANO}

Universidad Católica Argentina

distefanomj@gmail.com

https://orcid.org/0000-0002-0006-2303

* Financiamiento: El desarrollo de este proyecto fue posible gracias a un subsidio de la Templeton World Charity Foundation, Inc. y de la Universidad Austral otorgado a la Dra. Belén Mesurado. Las opiniones expresadas en este proyecto son de los investigadores y no necesariamente reflejan la visión de Templeton World Charity Fundación, Inc.

DOI: 10.15581/004.37.11-32 
Resumen: Las Tecnologías de la Información y la Comunicación (TIC) han generado grandes cambios en un sinfín de ámbitos, debido a que han posibilitado la introducción de tecnologías y la generación de nuevas metodologías de trabajo. En este contexto, Mesurado y colaboradores (2018) han desarrollado el Programa Héroe, primera aplicación web dirigida a promover conductas prosociales y variables socio-emocionales en adolescentes. Dado que Héroe es un desarrollo web reciente es de sumo interés indagar su usabilidad. De allí que los objetivos de este artículo sean 1). Estudiar la percepción de los usuarios en relación a la efectividad de cada uno de los módulos del programa Héroe en promover la prosocialidad, la empatía, la gratitud, el perdón y la satisfacción; y 2). Analizar las opiniones de los usuarios referidas a las características generales del programa, identificando las actividades que mayor nivel de agrado les generaron, como así también las que no les agradaron. La muestra estuvo compuesta por 158 adolescentes de 12 a 17 años ( $M=14.90$; $D E$ $=1.58,54 \%$ mujeres), de nivel socio-económico medio. Los resultados indican que existe un crecimiento irregular pero progresivo de cada una de las variables promovidas por el programa Héroe. Además, el programa tuvo un alto grado de aceptación por parte de los adolescentes que participaron de la intervención.

Palabras clave: Prosocialidad, Empatía, Gratitud, Perdón, Satisfacción, Usabilidad.
Abstract: Information and Communication Technologies (ITC) have brought about major changes in countless areas, as they have allowed for the inclusion of technology in education and have created new labor methodologies. In this context, Mesurado et al. (2018) have developed the online program Hero, the first web application aimed at promoting prosocial behavior and socio-emotional variables among adolescents. Given that the Hero program is new, investigating its usability is of great interest. Thus, the aims of this article are 1). To study users' perception of the effectiveness of each of the Hero program modules in the promotion of prosocial behavior, empathy, gratitude, forgiveness, and satisfaction; and 2). To analyze the users' opinions on the general characteristics of the program by identifying which activities they liked best and those which they did not like. The sample included 158 adolescents between the ages of 12 and 17 ( $M=14.90 ; \mathrm{DE}=1.58,54 \%$ women), from a middle socioeconomic status. The results indicate that there is an irregular but progressive growth in all variables promoted by the Hero program. Moreover, the program was highly acceptable to the adolescents who participated in the intervention.

Keywords: Prosocial behavior, Empathy, Gratitude, Forgiveness, Satisfaction, Usability.

\section{INTRODUCCIÓN}

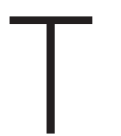

radicionalmente la conducta prosocial se describe como todo acto voluntario y positivo con intención de ayudar o beneficiar a otra persona o grupo (Eisenberg, Fabes y Spinrad, 2006). La conducta prosocial se distingue de la conducta de ayuda general en que busca mejorar la situación del destinatario y en que es siempre voluntaria (Bierhoff, 2002; Lam, 2013). Participar de actividades prosociales tiende a mejorar la percepción de sí mismo, y a desarrollar mejores habilidades sociales (Penner, Dovidio, Piliavin y Schroeder, 2005), favoreciendo la integración a la comunidad y la adaptación psicosocial en niños y adolescentes (Lam, 2013). Diversos estudios revelan que la prosocialidad favorece la toma de perspectiva (Stams, Brugman, Dekovic, van Rosmalen, van der Laan y Gibbs, 2006), la internalización de valores (Padilla-Walker y Fraser, 2014); e intensifica el sentido de transcendencia (Van Tongeren, Green, Davis, Hook y Hulsey, 2015). 
Por otro lado, disminuye la probabilidad del surgimiento de conductas desadaptativas e inhibe las conductas agresivas (Carlo, Crockett, Wilkinson y Beal, 2011; Padilla-Walker, Carlo y Nielson, 2015; Richaud, Mesurado y Kohan Cortada, 2012), el consumo de sustancias (Carlo et al., 2011), y la delincuencia (Padilla-Walker et al., 2015). Considerando la importancia que presentan las conductas prosociales para el desarrollo psicológico positivo, es un factor clave contar con programas cuyo objetivo primordial sea su promoción.

\section{Programa Héroe (Hero Program): estructura y Características}

Las Tecnologías de la Información y la Comunicación (TIC) han generado grandes cambios en un sinfín de ámbitos, debido a que han posibilitado la introducción de tecnologías y la generación de nuevas metodologías de trabajo. En este contexto, Mesurado, Distéfano, Robiolo y Richaud (2018) han desarrollado Héroe (Hero Program, www.herointervention.com), la primera aplicación web para promover las conductas prosociales en adolescentes. Esta herramienta se enmarca y toma las características de la era digital, es decir, que es autoadministrable: le permite estar disponible las veinticuatro horas del día, los siete días de la semana. Por otro lado, permite proliferarse ampliamente venciendo las barreras témporo-espaciales (Morris y Picard, 2014; Rose, 2014), alcanzando poblaciones en diversos sitios geográficos que sin la web no sería posible.

El desarrollo de Héroe se enmarcó en el campo de la Tecnología Positiva, definida como el uso de la tecnología para promover el desarrollo de fortalezas, el empoderamiento y el bienestar de las personas a nivel individual y social (Botella, Riva, Gaggioli, Wiederhold, Alcañiz y Baños, 2012; Villani, Cipresso, Gaggioli y Riva, 2016). La distribución en Internet de distintas intervenciones orientadas a incrementar el bienestar parece prometedora. Aunque sus efectos son modestos, existen estudios que comprueban su aceptación y efectividad, prescindiendo de entrenar profesionales para esos abordajes (Drozd, Mork, Nielsen, Raeder y Bjørkli, 2014; Schueller y Parks, 2012).

El programa Héroe está estructurado en cinco módulos o islas, tendientes a estimular variables socio-emocionales asociadas empíricamente con la prosocialidad: empatía y reconocimiento emocional, emociones positivas, gratitud y perdón (Mesurado et al., 2018). La estructura en sí se presenta como una aventura en la que el adolescente deberá navegar por distintas islas, que representan cada una de las variables y que cuentan con diversas actividades a realizar. La navegación entre las islas es lineal, es decir, que el joven debe seguir un recorrido preestablecido (empatía, gratitud, satisfacción, perdón y conducta prosocial) cumpliendo cada una de las 
actividades propuestas para poder proseguir. Está estudiado que el uso de diversas técnicas tiende a prolongar los efectos en el tiempo (Parks, Della Porta, Pierce, Zilca y Lyubomirsky, 2012). Por este motivo Héroe ofrece distintas herramientas de trabajo, algunas lúdicas, otras de relajación y reflexión, que contribuyen al cambio positivo del adolescente. Los recursos multimedia no sólo hacen la intervención más dinámica y atractiva, sino que también enriquecen y potencian las técnicas utilizadas, ofreciendo diversos canales de expresión (Barak, Klein y Proudfoot, 2009).

$\mathrm{Al}$ ingresar en la herramienta, los adolescentes deben elegir, entre tres posibles, un personaje que los represente en el programa. Luego son recibidos por un avatar con figura de maestro Sensei, que les presenta la intervención y los guía por el recorrido de las islas, mediante instrucciones y explicaciones en audio y texto. La presencia del maestro Sensei cumple la función de captar la atención y facilitar el seguimiento de las instrucciones por parte de los adolescentes (Hongpaisanwiwat y Lewis, 2003), mejorando la guía y la trazabilidad paulatina del avance de una isla a la siguiente. El programa cuenta con actividades multimedia que incorporaron diversos conceptos de gamification, como, por ejemplo, la barra de progresión, el puntaje, los premios o medallas, los avatares, junto con refuerzos positivos (tanto visuales como sonoros). La finalidad del uso de gamification es mantener la motivación de los adolescentes y su adherencia a la intervención hasta finalizarla (Cugelman, 2013; Kelders, Sommers-Spijkerman y Goldberg, 2018).

Cada módulo se realiza en sesiones independientes con una duración aproximada de 30 minutos cada uno, y no se pueden repetir. Cada isla comienza con un video psicoeducativo en el que se desarrolla y explica la variable a trabajar, a la vez que se busca motivar y comprometer al adolescente a reflexionar y profundizar en cada tema propuesto. Luego prosiguen distintas actividades para estimular cada variable.

A continuación se describen cada uno de los módulos o islas que componen el programa Héroe.

\section{Isla Empatía}

Este módulo comienza con un video psicoeducativo en el que se explica qué es la empatía y su relevancia en la vida social cotidiana, invitando a reflexionar acerca de la importancia de ponerse en el lugar del otro e identificar sus estados emocionales, en especial los de malestar y necesidad. Luego se presentan dos actividades para promover el reconocimiento emocional, habilidad importante e indispensable para poder dar respuestas empáticas (Baron-Cohen, 2011; Lawrence, Shaw, Baker, Baron-Cohen y David, 2004). En cada una de ellas se exhiben fotos estandarizadas con expresiones faciales de diversas emociones donde los adolescentes deben iden- 
tificar la emoción expresada (LoBue y Thrasher, 2015). Cada una de las actividades tiene una modalidad particular y, si bien están presentadas a modo de juego, su finalidad es de aprendizaje y entrenamiento, por lo que constan de múltiples intentos diferentes y, en caso de fallar, el sistema indica la respuesta correcta.

\section{Isla Gratitud}

Luego de un video psicoeducativo sobre la gratitud y sus implicancias personales y sociales, se presenta otro video en el que se observa un experimento sobre la gratitud expresada y sus efectos positivos. A continuación se ofrecen dos actividades para promover la gratitud como fortaleza personal (Emmons y Crumpler, 2000; Peterson y Seligman, 2004). Tomando como referencia las técnicas del Diario de Gratitud y el Álbum de Recuerdos Gratificantes (Emmons y McCullough, 2003; Lyubomirsk, 2008) se diseñó la primera actividad, que consiste en la elaboración de un Libro de Gratitud separado en capítulos según distintas etapas vitales. El adolescente es guiado en la reminiscencia de situaciones, personas o experiencias por los que pudiera estar agradecido para registrarlos en el capítulo correspondiente. La segunda actividad está basada en la técnica de la Carta de Agradecimiento (Seligman, 2002; Seligman, Steen, Park y Peterson, 2005) pero apoyada en recursos multimedia: invita a identificar una persona a la que se le está agradecido y a realizar un video expresándoselo. Finalizada cada actividad, se contará con la posibilidad de descargarlo para poder enviarlo al destinatario.

\section{Isla Satisfacción}

Las actividades de esta isla buscan suscitar emociones positivas y placenteras manipulando la cualidad emocional del estímulo que se presenta (Botella et al., 2012; Villani et al., 2016). La isla inicia con un video acerca de la importancia de las emociones positivas para el bienestar. Luego se da lugar a un ejercicio de relajación acompañado de imágenes y música calma (Baños et al., 2014), al que prosiguen dos actividades lúdicas que fomentan el foco en aspectos positivos (Wadlinger y Isaacowitz, 2008). La primera es una lluvia aleatoria de palabras positivas como amor, bondad, etc., y negativas como desesperanza, odio, etc., en la que el adolescente debe seleccionar con un clic las palabras positivas a la vez que va sumando puntos mientras evita las negativas. La última actividad consiste en una matriz de fotos con distintas emociones expresadas, entre las que el adolescente debe identificar la emoción positiva. A medida que avanza la matriz se va haciendo más compleja y va aumentando el desafío. 


\section{Isla Perdón}

Este módulo comienza con un video psicoeducativo en que se describe el perdón como un complejo proceso que beneficia al ofensor pero también, y principalmente, a quien perdona (Mooney, Strelan y McKee, 2016). La primera es una actividad de reflexión que invita a recordar alguna situación en la que se ha ofendido o lastimado a alguna persona y ésta le ha perdonado. Partiendo de la experimentación del perdón en primera persona, la segunda actividad propone al adolescente ejercitar él mismo el perdón. Se le propone que identifique alguna situación en la que él haya sido lastimado u ofendido, para luego trabajar en la resignificación positiva tanto del ofensor como de la situación (Karremans y Van Lange, 2008; Norman, 2017). Finalmente, se le invita a expresar su perdón (McCullough, 2008) mediante una carta que podrá decidir si la envía o la guarda.

\section{Isla Conducta Prosocial}

Esta última isla comienza con un video psicoeducativo en el que se describe la importancia del compromiso interpersonal y la solidaridad para el bienestar de toda la sociedad. Luego se sugiere un video debate en el que se reflexiona acerca del compromiso y sus implicaciones según se trate de acciones solidarias espontáneas o planificadas (Auné, Blum, Abal, Lozzia y Attorresi, 2014). Finalmente, se les ofrece a los adolescentes un listado de iniciativas solidarias concretas de entidades no gubernamentales de diversas zonas de su país. Se les brinda una descripción de la misma y sus datos de contacto, con el objetivo de fomentar el compromiso, las interrelaciones y la integración social mediante la generación de intenciones colectivas (Botella et al., 2012; Villani et al., 2016).

\section{USABILIDAD}

Los diferentes desarrollos tecnológicos han posibilitado que la creación, diseminación y el acceso a la información sea más rápido y más fluido. Dentro del vasto espacio de información actual, es sumamente necesario que un usuario pueda encontrar sólo la información relevante que satisface la necesidad de búsqueda original (Ball y Bothma, 2017). Las diversas herramientas software y aplicaciones, sean web, mobile o para wearables, deben estar alineadas con el concepto de usabilidad, definido por la ISO 25010. La usabilidad es entendida como el grado en el cual un producto o sistema puede ser utilizado por los usuarios para alcanzar los objetivos específicos con efectividad, eficiencia y satisfacción en un contexto de uso. 
Si una herramienta software o una aplicación no es fácil de utilizar muy probablemente quede en desuso, porque no ha cumplido el objetivo primordial de cualquier desarrollo: satisfacer las necesidades de los usuarios. Por lo cual, los desarrollos tecnológicos como el Programa Héroe requieren realizar diversas pruebas de usabilidad en pos de lograr las metas prosociales.

Dentro de las tecnologías con orientación prosocial, es viable mencionar la metodología propuesta por Dimitropoulos y sus colaboradores (2015), que abordan tres perspectivas en pos del análisis de una herramienta: i) la evaluación del rendimiento técnico de la plataforma y sus componentes, ii) la evaluación de usabilidad/aceptabilidad de la tecnología propuesta y iii) la eficacia científica de los juegos, que en el caso del programa Héroe sería la promoción de la prosocialidad.

En este contexto, y en pos de obtener una medición de la actitud de un grupo de estudio, Albaum (1997) y Chomeya (2010) sugieren la posibilidad de construir una escala de actitud de tipo Likert donde los participantes especifiquen su opinión y actitud para con la herramienta, indicando su nivel de acuerdo o desacuerdo con una declaración, ítem o pregunta. Por otro lado, dentro de los métodos de testeo de usabilidad con usuarios, Holzinger (2005) y Nielsen (1994) aconsejan abordar también el estudio de las características y funcionalidades preferidas por los usuarios.

\section{Nuestro estudio}

Dado que el programa Héroe fue desarrollado recientemente y contemplando una implementación masiva del mismo, es de sumo interés realizar una evaluación de su usabilidad. Para ello este artículo se plantea los siguientes objetivos:

1) Estudiar la percepción de los usuarios en relación a la efectividad de cada uno de los módulos del programa Héroe en promover la prosocialidad, la empatía, la gratitud, el perdón y la satisfacción.

Debido a que un gran número de estudios previos han mostrado que las variables incluidas en el programa son muy sensibles a las diferencias por sexo y edad (Álvarez Cabrera, Carrasco Gutiérrez y Fustos Mutis, 2010; Davila, Finkelstein y Castiene, 2011; Garaigordobil y Maganto, 2011) es fundamental controlar estas variables para evitar sesgos en los resultados obtenidos. Finalmente, nuestro segundo objetivo es:

2) Analizar las opiniones de los usuarios referidas a las características generales del programa, identificando las actividades que mayor nivel de agrado les generaron, así como también las que no les agradaron. 


\section{Metodología \\ Participantes y procedimiento}

La muestra estuvo compuesta por 158 adolescentes de 12 a 17 años $(M=14.90$; $\mathrm{DE}=1.58$ ); el $54 \%$ de los participantes fueron mujeres, de nivel socio-económico medio, todos residentes en Buenos Aires, Argentina.

El procedimiento utilizado para la selección de participantes se realizó desde una universidad argentina. Se publicitaron invitaciones a participar del estudio a adolescentes de entre 12 y 17 años, quienes deberían contar con la autorización de sus padres o tutores para participar del mismo. El primer paso consistió en la realización de una entrevista personal con los posibles participantes, en la cual se les explicó la finalidad del estudio y sus características (la cantidad de sesiones y la duración). También se les indicó que podían acceder al programa desde una página web habilitada para tal fin (www.herointervention.com), desde sus casas o bien desde algún lugar tranquilo que permitiera la reflexión personal. De la misma manera, se les indicó que para ello necesitaban contar con una computadora con cámara y sonido, así como con conexión a Internet.

Aquellos adolescentes que aceptaron participar en el programa debían firmar un consentimiento informado junto con sus padres, y recibían el código de acceso. El investigador obtiene el código de acceso automáticamente desde el administrador del programa para otorgárselo a los adolescentes. El participante necesita el código para poder generar un usuario y una contraseña personal para ingresar al programa. Todos los participantes de un grupo de intervención reciben el mismo código, resguardando así la confidencialidad de los datos. El código se utiliza con la intención de separar los grupos de intervención por períodos de tiempo u otras necesidades propias del diseño de investigación (ej. diferentes modalidades de implementación del Programa Héroe: desde el hogar o desde una institución educativa, etc.). Así mismo, el código de acceso se utiliza con la finalidad de que el programa no sea de libre acceso sino que puedan controlarse las habilitaciones desde el administrador. De este modo se garantiza la calidad de los datos registrados en el sistema.

Como se mencionó anteriormente, el programa consta de cinco módulos de intervención que estimulan la empatía, la gratitud, la satisfacción, el perdón y la conducta prosocial. Al finalizar cada módulo o isla se les solicitaba a los participantes que indicaran si las actividades realizadas les habían ayudado a estimular cada una de las variables promovidas en el programa. Los adolescentes recibían semanalmente un recordatorio por WhatsApp anunciando que estaba disponible la sesión siguiente. 
Una vez finalizada la realización de los cinco módulos se coordinó una segunda entrevista presencial con los participantes con la intención de indagar la usabilidad y sus opiniones acerca del programa.

\section{Instrumentos}

1) Evaluación al finalizar cada isla o módulo. Al finalizar cada uno de los módulos del programa se realizaron cinco preguntas desarrolladas ad boc para esta investigación con el objetivo de indagar la percepción de eficacia de los usuarios en la promoción de cada una de las variables trabajadas en el programa Héroe. Las cinco afirmaciones utilizadas fueron las siguientes: 1. La actividad me estimuló a querer ayudar a otras personas (Prosocialidad), 2. La actividad me ayudó a comprender a los demás (Empatía), 3. La actividad me ayudó a sentirme más agradecido con la vida (Gratitud), 4. En comparación a como me encontraba antes de realizar la actividad ahora me encuentro más feliz/contento (Satisfacción) y 5. La actividad me ayudó a querer perdonar a las personas que me han dañado (Perdón). Al finalizar cada isla, se les pidió a los participantes que indicaran su nivel de acuerdo para con cada una de las cinco afirmaciones, usando una escala de tipo Likert de 1 a 5 puntos, donde 1 significaba totalmente en desacuerdo y 5 totalmente de acuerdo.

2) Opiniones de los usuarios sobre el programa. Una vez finalizado el programa de intervención se convocó a los jóvenes a una entrevista personal dónde se les formularon tres preguntas abiertas: 1 . ¿Cuál es tu opinión general acerca del Programa Héroe?, 2. ¿Qué actividades te gustaron más del programa? y 3. ¿Qué actividades no te gustaron del programa? Las entrevistas fueron grabadas, tipeadas y posteriormente codificadas por dos investigadores.

\section{Procedimiento estadístico}

Con la intención de abordar el primer objetivo del estudio se realizaron cinco ANCOVA de medidas repetidas analizando las variaciones de los niveles de prosocialidad, empatía, gratitud, perdón y satisfacción a lo largo de los 5 módulos que componen el programa, controlando el sexo y la edad de los participantes. Para abordar el segundo objetivo del estudio, primero se codificaron las respuestas de los participantes y luego se utilizaron estadísticas descriptivas para el análisis. 


\section{RESULTADOS}

Modificación de la percepción de prosocialidad, empatía, gratitud, perdón y satisfacción a lo largo de los 5 módulos de los participantes

Con el objetivo de estudiar el cambio en los niveles de la percepción de eficacia de cada uno de los módulos que componen el programa se realizaron 5 ANCOVAS de medidas repetidas para cada una de las variables controlando el sexo y la edad de los participantes.

\section{Empatía}

El ANCOVA de medidas repetidas indicó que cada uno de los módulos que componen la intervención promovían positivamente la percepción de empatía entre los participantes [Traza de Hotelling F $(4,123)=9.68, p \leq .001$, eta $=.24]$. No se halló ningún efecto en relación al sexo [Traza de Hotelling $\mathrm{F}(4,123)=1.85, \mathrm{p}=.12$, eta $=.06$ ] ni a la edad de los participantes [Traza de Hotelling $\mathrm{F}(20,486)=1.39, \mathrm{p}=$ .12 , eta $=.05]$.

\section{Gratitud}

El ANCOVA de medidas repetidas indicó que cada uno de los módulos que componen la intervención promovieron positivamente la percepción de los niveles de agradecimiento entre los participantes [Traza de Hotelling $\mathrm{F}(4,123)=20.35, p$ $\leq$. 001, eta $=.40]$. Por otro lado, también se encontraron diferencias significativas en los niveles de percepción de gratitud en función de la edad de los participantes [Traza de Hotelling F $(20,486)=1.69, p \leq .-.03$, eta $=.06$ ]. No se halló ningún efecto en relación al sexo [Traza de Hotelling $\mathrm{F}(4,123)=1.88, \mathrm{p}=.12$, eta $=.06$ ]

\section{Satisfacción}

El ANCOVA de medidas repetidas indicó que cada uno de los módulos que componen la intervención promovieron positivamente la percepción de satisfacción entre los participantes [Traza de Hotelling F $(4,123)=14.76, p \leq .001$, eta $=.32$ ] . No se halló ningún efecto en relación al sexo [Traza de Hotelling F $(4,123)=.81$, $\mathrm{p}=.52$, eta $=.03$ ] ni a la edad de los participantes [Traza de Hotelling F $(20,486)$ $=.77, \mathrm{p}=.75$, eta $=.03]$. 


\section{Perdón}

El ANCOVA de medidas repetidas indicó que cada uno de los módulos que componen la intervención promovió positivamente la percepción de su capacidad de perdonar entre los participantes [Traza de Hotelling F $(4,123)=15.54, p \leq$. 001, eta $=.34$ ]. No se halló ningún efecto en relación al sexo [Traza de Hotelling $\mathrm{F}$ (4, $123)=.51, \mathrm{p}=.73$, eta $=.02$ ] ni a la edad de los participantes [Traza de Hotelling $\mathrm{F}$ $(20,486)=.99, \mathrm{p}=.71$, eta $=.03]$.

\section{Prosocialidad}

Por último, el ANCOVA de medidas repetidas indicó que cada uno de los módulos que componen la intervención promovió positivamente la percepción de prosocialidad entre los participantes [Traza de Hotelling F $(4,123)=22.67, p \leq .001$, eta $=$ .43]. No se halló ningún efecto en relación al sexo [Traza de Hotelling F $(4,123)$ $=.33, \mathrm{p}=.86$, eta $=.01$ ] ni a la edad de los participantes [Traza de Hotelling F (20, 486) $=1.03, \mathrm{p}=.43$, eta $=.04]$.

La Figura 1 muestra la trayectoria de los puntajes de la percepción de empatía, gratitud, satisfacción, perdón y prosocialidad de los usuarios en cada uno de los módulos a lo largo de la intervención del programa Héroe. Como puede observarse, existe un crecimiento irregular pero progresivo de cada una de las variables promovidas por el programa Héroe.

Figura 1. Trayectoria de los puntajes de la percepción de los niveles de empatía, gratitud, satisfacción, perdón y prosocialidad de los participantes en cada módulo que compone el Programa Héroe

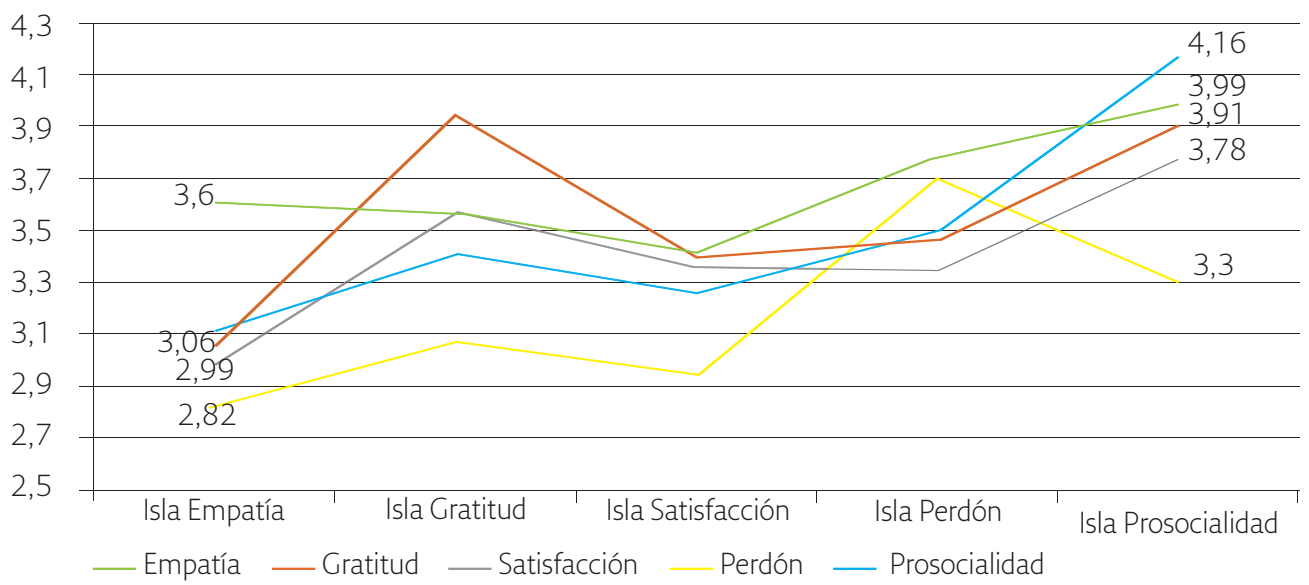


Opiniones de los usuarios sobre el Programa Héroe

Una vez finalizada la intervención se convocó a los 158 adolescentes a una entrevista personal. El 39\% de los participantes $(n=61)$ asistieron a la entrevista final. Con base en la información recogida de esas respuestas se realizaron los siguientes análisis.

Con el objetivo de conocer la opinión de los usuarios del programa se codificaron las respuestas espontáneas obtenidas en las entrevistas personales que se mantuvieron con los adolescentes. La Tabla 1 muestra la frecuencia y los porcentajes de la evaluación general que hicieron los adolescentes acerca de los aspectos generales del programa. Como puede verse, el 44\% de los participantes valoran positivamente el formato de la actividad, dicen que las actividades son interesantes, originales, creativas y entretenidas. En segundo lugar, el 31\% de los adolescentes interpretan que las actividades del programa les ayudaron a reflexionar sobre sus propias vidas, sobre sus actitudes, a repensar sobre temas en los que no suelen pensar cotidianamente y a reflexionar sobre los principales valores de la vida. Por otra parte sorprende que sólo el $4 \%$ de los adolescentes hayan indicado espontáneamente que lo aprendido en el programa pueden transferirlo a la vida cotidiana. Sería interesante en futuros estudios evaluar este aspecto consultando a los adolescentes específicamente acerca de este punto, con la intención de obtener una opinión de todos los participantes sobre la posible transferencia a la vida cotidiana de lo aprendido en Héroe. Ver Tabla 1.

Tabla 1. Aspectos generales del programa más valorados espontáneamente por los usuarios

ASPECTOS GENERALES DEL PROGRAMA

FRECUENCIA

PORCENTAJE

\begin{tabular}{lll}
\hline 1. Características del programa (formato, creatividad, entretenido, originalidad) & 35 & $44 \%$ \\
\hline 2. Reflexión (valores, temas personales) & 25 & $31 \%$ \\
\hline 3. Diversidad de Actividades & 6 & $8 \%$ \\
\hline 4. Temas tratados & 5 & $6 \%$ \\
\hline 5. Guía - Sensei & 4 & $5 \%$ \\
\hline 6. Transferir lo aprendido a mi vida cotidiana & 3 & $4 \%$ \\
\hline 7. Sistema de premios & 2 & $3 \%$ \\
\hline
\end{tabular}

Seguidamente se codificaron y analizaron las respuestas espontáneas que dieron los adolescentes referidas a qué actividades les gustaron más durante la intervención Héroe. Los resultados que pueden verse en la Tabla 2 indican que las actividades más valoradas por los adolescentes fueron en un 44\% los videos 
que están incluidos a lo largo del todo el programa como introducción a cada una de las islas que componen el mundo Héroe, así como el uso de los videos como disparadores de las actividades (Isla Gratitud) y el video debate incluido en la Isla Compromiso. En segundo lugar, las actividades contenidas en la Isla Empatía fueron las más preferidas por los adolescentes en un 14\%, seguidas de las preguntas contenidas en el programa por un $11 \%$ y las actividades de la Isla Compromiso en un $10 \%$.

Tabla 2. Actividades del programa más valorados por los usuarios

\begin{tabular}{lcc} 
QUÉ ACTIVIDAD TE GUSTÓ MÁS & FRECUENCIA & PORCENTAIE \\
\hline 1. Videos & 28 & $44 \%$ \\
\hline 2. Actividades de la Isla Empatía & 9 & $14 \%$ \\
\hline 3. Preguntas contenidas en el programa & 7 & $11 \%$ \\
\hline 4 4. Actividades Isla Compromiso & 6 & $10 \%$ \\
\hline 5. Actividades Isla Perdón & 5 & $8 \%$ \\
\hline 6. Actividades Isla Gratitud & 4 & $6 \%$ \\
\hline 7. Actividades Isla Satisfacción & 4 & $6 \%$ \\
\hline
\end{tabular}

Por último, se analizaron los aspectos menos valorados por los usuarios en el programa. En la Tabla 3 puede observarse que el $8 \%$ de los entrevistados indicaron que les habían gustado todos los aspectos del programa y que por ello no podían indicar nada que no les hubiese gustado. Entre los aspectos menos valorados del programa, el $22 \%$ de los adolescentes indicó que las preguntas que contenía el programa para valorar las variables fueron extensas y reiterativas, el 20\% de los participantes informó haber tenido algún tipo de problema técnico, como por ejemplo lentitud en la conexión a Internet, lentitud en el pasaje de una actividad a otra, o bien errores que surgieron durante la ejecución del programa debido a problemas técnicos en su ordenador o bien de la conexión a Internet. Entre las actividades realizadas dentro del programa, la actividad que menos gustó (11\% de los participantes) fue la de filmar un video expresando su agradecimiento a alguna persona. Aunque se les había aclarado que el contenido de la filmación era privado y los investigadores no tendríamos acceso a esa información, los principales motivos del desagrado de la actividad fueron por vergüenza o incomodidad con la actividad. A un 11\% de los participantes les pareció excesiva la extensión de la intervención. 
Tabla 3. Actividades del programa más valoradas por los usuarios

\begin{tabular}{lcc} 
ASPECTOS QUE NO GUSTARON DEL PROGRAMA & FRECUENCIA & PORCENTAJE \\
\hline 1. Preguntas (extensión, reiterativo) & 14 & $22 \%$ \\
\hline 2. Problemas técnicos (lentitud, errores) & 13 & $20 \%$ \\
\hline 3. Filmar el video (actividad Isla Gratitud) & 7 & $11 \%$ \\
\hline 4. La extensión de la intervención & 7 & $11 \%$ \\
\hline 5. Indicaron que les gustó todo & 5 & $8 \%$ \\
\hline 6. Videos (tipo, extensión) & 3 & $5 \%$ \\
\hline 7. El personaje que guía la intervención (Sensei) & 3 & $5 \%$ \\
\hline 8. Gráfica & 3 & $5 \%$ \\
\hline 9. Escribir (actividades Isla Gratitud, Perdón, Compromiso) & 2 & $3 \%$ \\
\hline 10. Aburrido & 2 & $3 \%$ \\
\hline 11. Actividades Isla Perdón & 1 & $1.5 \%$ \\
\hline 12. Actividades repetitivas & $1.5 \%$ \\
\hline 13. No poder personalizar el avatar & 1 & $1.5 \%$ \\
\hline 14. Actividades de Isla Empatía & 1 & $1.5 \%$ \\
\hline 15. No comprender la utilidad & 1 & $1.5 \%$ \\
\hline
\end{tabular}

\section{DisCUSIÓN}

El primer objetivo de esta investigación fue estudiar la percepción de los adolescentes en relación a la efectividad de cada uno de los módulos del programa Héroe a la hora de promover la percepción de los usuarios de su nivel de prosocialidad, empatía, gratitud, perdón y emociones positivas (ej. relajación, disfrute, entre otras). Como muestra la Figura 1, no hay un crecimiento lineal secuencial en cada una de las variables de una sesión a otra, sino que el crecimiento es irregular. Sin embargo, si se compara el puntaje de la sesión inicial y final de cada variable, hay un incremento en todas las variables percibidas por los participantes. Por otro lado, es importante resaltar que la percepción de los adolescentes de su propio nivel de gratitud, perdón y prosocialidad puntúa más alto en aquellas sesiones en las que se estimuló específicamente esa variable socio-emocional, a excepción de la percepción de empatía y satisfacción, donde su mayor puntaje en promedio fue obtenido en la última sesión de la intervención. Esto podría deberse a un efecto acumulativo del programa de intervención. Así mismo, debe tenerse en cuenta que todas estas variables socio-emocionales estimuladas en el programa Héroe están relacionadas unas con otras; consecuentemente, es pro- 
bable que cuando el programa de intervención promueve una de ellas, indirectamente está promoviendo las demás.

Estos resultados serían un primer indicio de que las actividades incluidas en el programa parecen cumplir con el objetivo de promover la percepción de los usuarios de sus niveles de prosocialidad, así como cada una de las variables vinculadas empíricamente a ella, como la percepción de empatía, gratitud, satisfacción y perdón. Por otro lado, es importante resaltar que es probable que la intervención tenga un efecto acumulativo, dado que las actividades de cada módulo o isla parecen ir reforzando la estimulación de las variables incluidas en el programa, de allí su efecto creciente entre la primera sesión y la última.

Estudios previos revelaron que las mujeres presentan una mayor tendencia hacia las conductas prosociales (Álvarez Cabrera, Carrasco Gutiérrez y Fustos Mutis, 2010; Davila, Finkelstein y Castiene, 2011; Tur-Porcar, Llorca, Malonda, Samper y Mestre, 2016), así como la empatía predomina en las mujeres de distintas edades más que en los varones (Garaigordobil y García de Galdeano, 2006; Garaigordobil y Maganto, 2011; Sánchez-Queija, Oliva y Parra, 2006; Oros y Fontana, 2013). Sin embargo, nuestros resultados no lograron evidenciar diferencias en la percepción de eficacia del programa entre los varones y las mujeres de diferentes edades que participaron en el programa Héroe.

Respecto a la expresión de gratitud, algunos estudios previos no han encontrado diferencias significativas entre niños varones y mujeres en Brasil y Estados Unidos (de Lucca Freitas, Pieta y Tudge, 2011; Froh, Yurkewicz y Kashdan, 2009); sin embargo, un estudio con niños argentinos de entre 9 y 13 años reveló puntajes significativamente mayores en las niñas (Cuello y Oros, 2016). Nuestros resultados estuvieron en consonancia con los estudios previos desarrollados en Brasil y Estados Unidos, puesto que no hallaron diferencias en la eficacia de la intervención en función del sexo pero sí en función de la edad de los adolescentes. Los adolescentes mayores en edad presentaban un mayor nivel de percepción de gratitud que los más jóvenes en el transcurso de la intervención, lo que puede deberse a un incremento de la capacidad reflexiva.

Por otro lado, debido a que estudios previos sobre emociones positivas han tenido en cuenta la diferencia entre los sexos, en nuestros estudios hemos controlado esta variable (Richaud y Mesurado, 2016). Asimismo, Miller, Worthington y McDaniel (2008) han encontrado que las mujeres son algo más indulgentes que los varones (efecto pequeño a moderado), aunque en general los estudios no revelan diferencias significativas en el perdón según el sexo (Fehr, Gelfand y Nag, 2010; Rana, Meena, Durgesh y Kallavarapu, 2014; Toussaint y Webb, 2005). Siguiendo con esta línea, en nuestro estudio no se encontraron diferencias ni de la edad ni del 
sexo en la percepción de eficacia de los módulos que componen la intervención en relación a la satisfacción (entendida como una emoción positiva), ni en relación a la variable percepción de perdón.

En síntesis, podemos afirmar que, si bien existen algunos estudios que encontraron diferencias en prosocialidad, empatía, gratitud, satisfacción y perdón en función de la edad o del sexo (Álvarez Cabrera, Carrasco Gutiérrez y Fustos Mutis, 2010; Davila, Finkelstein y Castiene, 2011; Garaigordobil y Maganto, 2011), nuestro estudio ha mostrado que la percepción de eficacia de los diferentes módulos que componen el programa Hero se han mostrado invariantes en relación a estas variables, con excepción de la gratitud. Es importante resaltar que los estudios arriba mencionados evaluaron las variables utilizando auto-informes, mientras que en este estudio se evaluó la auto-percepción de los adolescentes a través de ítems únicos, lo cual podría ser un factor diferencial importante. Sin embargo, pensamos que nuestros resultados son alentadores dado que los efectos del programa se mantendrían constantes en los adolescentes independientemente de estas características.

El segundo objetivo de este estudio era analizar las opiniones de los usuarios referidas a las características generales del programa, identificar las actividades que más les agradaron y las que menos les agradaron.

A partir de los resultados podemos afirmar que, en general, el programa tuvo un alto grado de aceptación por parte de los adolescentes que participaron de la intervención. Los puntos indicados como más significativos fueron las características del programa, resaltando el formato, la creatividad de las actividades, la originalidad de las mismas, así como lo entretenido que fue para ellos seguir las consignas propuestas por el Sensei. Estos aspectos son fundamentales para generar mayor adherencia a la intervención y promover la motivación de los adolescentes en el programa, dado que no está presente un profesional en las sesiones. Otro de los aspectos generales del programa resaltados por los adolescentes fue su capacidad de guiarlos en un camino de reflexión personal. Que los participantes puedan identificar este punto como un aspecto característico de Héroe indica una importante fortaleza del programa, dado que favorecer la observación y la reflexión personal es un elemento central en la promoción de cualquier fortaleza del carácter.

Entre los aspectos más valorados del programa los adolescentes mencionaron el uso de los videos como recursos de formación, educación, reflexión e inspiración de ideas para lograr la acción prosocial. Recurso que fue utilizado a lo largo de toda la intervención. Entre los aspectos menos valorados, los adolescentes resaltaron la reiteración y extensión de preguntas para evaluar la eficacia de la intervención, así como el surgimiento de diferentes problemas técnicos en el uso del programa. Algunos de 
estos problemas técnicos vienen vinculados con la calidad de los recursos de los que dispone el adolescente (conexión a Internet o capacidad de su computadora, entre otros); sin embargo, otros pueden estar más vinculados a las características técnicas propias del programa. Esta información resulta sumamente valiosa para el equipo de investigación, dado que tendremos que hacer una detallada revisión técnica del programa Héroe para agilizar el uso de la herramienta informática y para que no sea un elemento que pueda desalentar a los adolescentes en el uso del mismo.

\section{Limitaciones y futuros estudios}

Una de las limitaciones más importante de este estudio fue la pérdida de participantes a la hora de realizar la entrevista final con los jóvenes. Sólo el 39\% de los participantes accedieron a asistir, a pesar de convocarlos a través de diferentes vías de comunicación y en diferentes ocasiones. Es probable que contar con la totalidad de los adolescentes en esta fase del estudio nos hubiera brindado mayor información sobre los aspectos analizados, y así nos permitiría realizar una revisión más profunda y completa tanto de los aspectos positivos como negativos del programa Héroe.

A partir de los resultados obtenidos podemos concluir que los niveles de aceptación y usabilidad del programa, así como la percepción subjetiva de los usuarios sobre la eficacia de los módulos del programa, fueron satisfactorios. De ahí que en el futuro sea necesario implementar el programa estudiando la eficacia pretest, post-test y de seguimiento en una amplia muestra de adolescentes. Es decir, cumplimentado el estudio de usabilidad de una manera satisfactoria, es necesario estudiar ahora en qué medida el programa es eficaz para promover la prosocialidad, la empatía, la gratitud, el perdón y la satisfacción en adolescentes de ambos sexos y de estas edades. Así mismo, en futuros estudios sería necesaria la implementación de otros procesos de evaluación de las variables más estandarizados y fiables, como el uso de auto-informes, reportes de pares, docentes o tutores.

Fecha de recepción del original: 24 de octubre 2018

Fecha de aceptación de la versión definitiva: 25 de febrero 2019

\section{REFERENCIAS}

Albaum, G. (1997). The Likert scale revisited. International fournal of Market Research, 39(2), 331-348.

Álvarez Cabrera, P., Carrasco Gutiérrez, M. y Fustos Mutis, J. (2010). Relación de la empatía y género en la conducta prosocial y agresiva, en adolescentes 
de distintos tipo de establecimientos educacionales. Revista Iberoamericana de Psicología: Ciencia y Tecnología, 3(2), 27-36.

Auné, S., Blum, D., Abal, F. J., Lozzia, G. S. y Atorresi, H. F. (2014). La conducta prosocial: Estado actual de la investigación. Perspectivas en Psicología: Revista de Psicología y Ciencias Afines, 11(2), 21-33.

Ball, L. H. y Bothma, T. J. D. (2017). The importance of usability evaluation when developing digital tools for a library-a case study. In Improving Quality of Life Through Information. Proceedings of the XXV Bobcatsss Symposium, Tampere, Finlandia.

Baños, R. M., Etchemendy, E., Farfallini, L., García-Palacios, A., Quero, S. y Botella, C. (2014). EARTH of well-being system: A pilot study of an information and communication technology-based positive psychology intervention. The Journal of Positive Psychology, 9(6), 482-488.

Barak, A., Klein, B. y Proudfoot, J. G. (2009). Defining internet- supported therapeutic interventions. Annals of Behavioral Medicine, 38, 4-17.

Baron-Cohen, S. (2011). The science of evil: On empathy and the origins of cruelty. New York: Basic Books.

Bierhoff, H. W. (2002). Prosocial behaviour. New York: Psychology Press.

Botella, C., Riva, G., Gaggioli, A., Wiederhold, B. K., Alcañiz, M. y Baños, R. M. (2012). The Present and Future of Positive Technologies. Cyberpsychology, behavior and Social Networking, 15(2), 1-7.

Carlo, G., Crockett, L. J., Wilkinson, J. L. y Beal, S. J. (2011). The longitudinal relationships between rural adolescents' prosocial behaviors and young adult substance use. Fournal of Youth and Adolescence, 40(9), 1192-1202.

Chomeya, R. (2010). Quality of psychology test between Likert scale 5 and 6 points. Fournal of Social Sciences, 6(3), 399-403.

Cuello, M. y Oros, L. B. (2016). Construcción de una escala para medir gratitud en niños de 9 a 12 años. Revista de Psicología clínica con niños y adolescentes, 3, 35-41.

Cugelman, B. (2013). Gamification: what it is and why it matters to digital health behavior change developers. 7MIR Serious Games, 1(1): e3.

Dávila, M. C., Finkelstein, M. A. y Castien, J. I. (2011). Diferencias de género en conducta prosocial: el comportamiento de ciudadanía organizacional. Psicothema, 23(3), 401-406.

Dimitropoulos, K., Vuillier, L., Modefferi, S., Middleton, L., Peters, C., d'Andria, F., Zoakou, A., Star, K., Thermos, S., Kaza, K., Psaltis, A., Stefanidis, K., Apostolakis, K. y Daras, P. (2015). ProsocialLearn: D2. 5 evaluation strategy and protocols. Extraído el 7 de febrero de 2019 de https://eprints.soton. ac.uk/386601/1/386601.pdf 
Drozd, F., Mork, L., Nielsen, B., Raeder, S. y Bjørkli, C. A. (2014). Better days-A randomized controlled trial of an internet-based positive psychology intervention. The Fournal of Positive Psychology, 9(5), 377-388.

Eisenberg, N., Fabes, R. A. y Spinard, T. L. (2006). Prosocial Development. En N. Eisenberg, W. Damon y R. M. Lerner (Eds.), Handbook of child psychology: Social, emotional, and personality development (pp. 646-718). Hoboken: John Wiley and Sons.

Emmons, R. A. y Crumpler, C. A. (2000). Gratitude as a Human Strength: Appraising the Evidence. Journal of Social and Clinical Psychology, 19(1), 56-69.

Emmons, R. A. y McCullough, M. E. (2003). Counting Blessings Versus Burdens: An Experimental Investigation of Gratitude and Subjective Well-Being in Daily Life. Fournal of Personality and Social Psychology, 84(2), 377-389.

Fehr, R., Gelfand, M. J. y Nag, M. (2010). The road to forgiveness: A meta-analytic synthesis of its situational and dispositional correlates. Psychological Bulletin, 136(5), 894-914.

de Lucca Freitas, L. B., Pieta, M. A. M. y Tudge, J. R. H. (2011). Beyond politeness: the expression of gratitude in children and adolescents. Psicologia: Reflexão e Crítica, 24(4), 757-764.

Froh, J. J., Yurkewicz, C. y Kashdan, T. B. (2009). Gratitude and subjective wellbeing in early adolescence: Examining gender differences. Fournal of adolescence, 32(3), 633-650.

Garaigordobil, M. y García de Galdeano, P. (2006). Empatía en niños de 10 a 12 años. Psicothema, 18(2), 180-186.

Garaigordobil, M. y Maganto, C. (2011). Empatía y resolución de conflictos durante la infancia y la adolescencia. Revista Latinoamericana de Psicología, 43(2), 255-266.

Holzinger, A. (2005). Usability engineering methods for software developers. Communications of the ACM, 48(1), 71-74.

Hongpaisanwiwat, C. y Lewis, M. (2003). The effect of animated character in multimedia presentation: Attention and comprehension. Proceedings of the IEEE International Conference on Systems, Man and Cybernetics, 2, 1350-1352.

Karremans, J. C. y Van Lange, P. A. (2008). Forgiveness in personal relationships: Its malleability and powerful consequences. European Review of Social Psycho$\log y, 19,202-241$.

Kelders, S. M., Sommers-Spijkerman, M. y Goldberg, J. (2018). Investigating the Direct Impact of a Gamified Versus Nongamified Well-Being Intervention: An Exploratory Experiment. Fournal of Medical Internet Research, 20(7): e247. 
Lam, C. M. (2013). Prosocial involvement as positive youth development construct: A conceptual review. Fournal of Alternative Medical Research, 5(1), 9-18.

Lawrence, E. J., Shaw, P., Baker, D., Baron-Cohen, S. y David, S. (2004). Measuring empathy: Reliability and validity of the empathy quotient. Psychological Medicine, 34, 911-924.

LoBue, V. y Thrasher, C. (2015). The Child Affective Facial Expression (CAFE) set: Validity and reliability from untrained adults. Frontiers in Psychology, 5, 1532 .

Lyubomirsky, S. (2008). La ciencia de la felicidad. Barcelona: Urano.

McCullough, M. E. (2008). Beyond revenge: The evolution of the forgiveness instinct. San Francisco: Jossey-Bass.

Mesurado, B., Distéfano, M. J., Robiolo, Y. y Richaud, M. C. (2018). The Hero program: Development and initial validation of an intervention program to promote prosocial behavior in adolescents. Fournal of Social and Personal Relationships https://doi.org/10.1177/0265407518793224

Miller, A. J., Worthington, E. L., Jr. y McDaniel, M. A. (2008). Gender and forgiveness: A meta-analytic review and research agenda. Fournal of Social and Clinical Psychology, 27(8), 843-876.

Mooney, L., Strelan, P. y McKee, I. (2016). How forgiveness promotes offender pro-relational intentions: The mediating role of offender gratitude. British Fournal of Social Psychology, 55(1), 44-64.

Morris, R. R. y Picard, R. (2014). Crowd-powered Positive Psychological Interventions. The Fournal of Positive Psychology, 9(6), 509-516.

Nielsen, J. (1994). Usability Inspection Methods. Trabajo presentado en la Conference Companion "Celebrating Interdependence", Boston, 24-28 de abril. http://www.idemployee.id.tue.nl/g.w.m.rauterberg/lecturenotes/0h420/niel$\operatorname{sen}[1994] . p d f$

Norman, K. (2017). Forgiveness: How it manifests in our health, well-being, and longevity. Master of Applied Positive Psychology (MAPP) Capstone Projects. University of Pennsylvania Scholarly Commons.

Oros, L. B. y Fontana, A. (2013). Los beneficios sociales de la empatía y de las emociones positivas durante la niñez. Trabajo presentado en el XXXIV Congreso Interamericano de Psicología. Brasilia.

Padilla Walker, L. M., Carlo, G. y Nielson, M. G. (2015). Does helping keep teens protected? Longitudinal bidirectional relations between prosocial behavior and problem behavior. Child Development, 86(6), 1759-1772.

Padilla-Walker, L. M. y Fraser, A. M. (2014). How much is it going to cost me? 
Bidirectional relations between adolescents' moral personality and prosocial behavior. Fournal of Adolescence, 37(7), 993-1001.

Parks, A. C., Della Porta, M. D., Pierce, R. S., Zilca, R. y Lyubomirsky, S. (2012). Pursuing happiness in everyday life: The characteristics and behaviors of online happiness seekers. Emotion, 12, 1222-1234.

Penner, L. A., Dovidio, J. E., Piliavin, J. A. y Schroeder, D. A. (2005). Prosocial behaviour: Multilevel perspective. Annual review of psychology, 56(1), 365-92.

Peterson, C. y Seligman, M. E. P. (2004). Character strengths and virtues: A classification and handbook. Washington: American Psychological Association/New York: Oxford University Press.

Rana, S., Meena, H., Durgesh, N. y Kallavarapu, V. (2014). Forgiveness: A determinant of adolescents' happiness. Indian Fournal of Health y Wellbeing, 5(9), $1119-1123$.

Richaud, M. C. y Mesurado, B. (2016). Las emociones positivas y la empatía como promotores de las conductas prosociales e inhibidores de las conductas agresivas. Acción Psicológica, 13(2), 31-42.

Richaud, M. C., Mesurado, B. y Kohan Cortada, A. (2012). Analysis of dimensions of prosocial behavior in an Argentinean sample of children. Psychological Reports: Mental y Physical Health, 111(3), 687-696.

Rose, R. D. (2014). Self-guided multimedia stress management and resilience training. The fournal of Positive Psychology, 9(6), 489-493.

Sánchez-Queija, L., Oliva, A. y Parra, A. (2006). Empatía y conducta prosocial durante la adolescencia. Revista de Psicología Social, 21(3), 259-271.

Schueller, S. M. y Parks, A. C. (2012). Disseminating self-help: Positive psychology exercises in an online trial. Fournal of medical Internet Research, 14, e63.

Seligman, M. E. P. (2002). La auténtica felicidad. Barcelona: Ediciones B.

Seligman, M. E. P., Steen, T. A., Park, N. y Peterson, C. (2005). Positive Psychology Progress: Empirical Validation of Interventions. American Psychologist, 60(5), 410-421.

Stams, G. J. M. M., Brugman, D., Dekovic, M., van Rosmalen, L., van der Laan, P. y Gibbs, J. C. (2006). The moral judgment of juvenile delinquents: A metaanalysis. Fournal of Abnormal Child Psychology, 34(5), 697-713.

Toussaint, L. y Webb, J. R. (2005). Gender Differences in the Relationship between Empathy and Forgiveness. Fournal of Social Psychology, 145(6), 673-685.

Tur-Porcar, A., Llorca, A., Malonda, E., Samper, P. y Mestre, M. V. (2016). Empatía en la adolescencia. Relaciones con razonamiento moral prosocial, conducta prosocial y agresividad. Acción Psicológica, 13(2), 3-14. 
Van Tongeren, D. R., Green, J. D., Davis, D. E., Hook, J. N. y Hulsey, T. L. (2015). Prosociality enhances meaning in life. The Fournal of Positive Psycholo$g y, 11(3), 225-236$.

Villani, D., Cipresso, P., Gaggioli, A. y Riva. G. (Eds.) (2016). Integrating Technology in Positive Psychology. Hershey: IGI Global.

Wadlinger, H. A. y Isaacowitz, D. M. (2008). Looking happy: The experimental manipulation of a positive visual attention bias. Emotion, 8, 121-126. 